Philipp Weber

Kosmos und Subjektivität in der Frühromantik 

Philipp Weber

\section{Kosmos und \\ Subjektivität in der Frühromantik}

Wilhelm Fink 
Bibliografische Information der Deutschen Nationalbibliothek

Die Deutsche Nationalbibliothek verzeichnet diese Publikation in der Deutschen Nationalbibliografie; detaillierte bibliografische Daten sind im Internet über http://dnb.d-nb.de abrufbar.

Alle Rechte vorbehalten. Dieses Werk sowie einzelne Teile desselben sind urheberrechtlich geschützt. Jede Verwertung in anderen als den gesetzlich zugelassenen Fällen ist ohne vorherige schriftliche Zustimmung des Verlags nicht zulässig.

(C) 2017 Wilhelm Fink Verlag, ein Imprint der Brill-Gruppe

(Koninklijke Brill NV, Leiden, Niederlande; Brill USA Inc., Boston MA, USA; Brill Asia Pte Ltd, Singapore; Brill Deutschland GmbH, Paderborn, Deutschland)

Internet: www.fink.de

Einbandgestaltung: Evelyn Ziegler, München Herstellung: Brill Deutschland GmbH, Paderborn

ISBN 978-3-7705-5908-4 


\section{Inhalt}

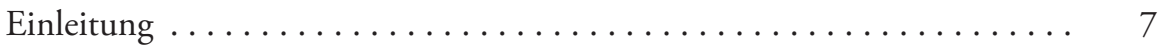

I. ROMANTISCHE KOSMOLOGIE $\ldots \ldots \ldots \ldots \ldots \ldots \ldots \ldots, 25$

Destruktion des Kosmos ............................. 27

Bewusstsein des Unendlichen . . . . . . . . . . . . . . . 47

Polyhymnios Schweigen ....................... 65

II. EPISTEMISCHER KOPERNIKANISMUS $\ldots \ldots \ldots \ldots \ldots \ldots$

Vielheit der Perspektiven ......................... 101

Universalnexus ........................... 123

III. INVERSION DES BLICKS $\ldots \ldots \ldots \ldots \ldots \ldots \ldots \ldots \ldots \ldots$

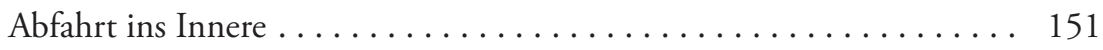

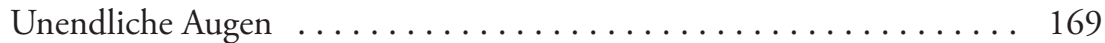

Grundlose Nacht . . . . . . . . . . . . . . . . . . . . . . 193

Literaturverzeichnis . . . . . . . . . . . . . . . . . . 213

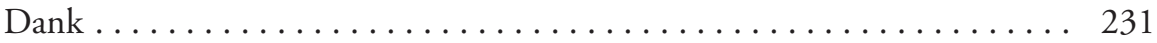

Ausführliches Inhaltsverzeichnis $\ldots \ldots \ldots \ldots \ldots \ldots \ldots \ldots \ldots \ldots \ldots \ldots \ldots$ 



\section{Einleitung}

Aufgabe der vorliegenden Untersuchung ist die Analyse des Zusammenhangs von Kosmos und Subjektivität in der Frühromantik. Kosmos und Subjektivität - dieses Begriffspaar stellt sogleich einen Antagonismus vor, denn Subjektivität konstituiert sich alleine im irreduziblen Bruch mit der kosmischen Einheit. Gegen Ende des 18. Jahrhunderts kommt es zu diesem Bruch, der sich durch ein Ineinanderwirken von wissenschaftlichen, philosophischen und ästhetischen Diskursen auszeichnet. Das Ende der traditionellen Vorstellung des Kosmos bedeutet nicht nur eine Revolution der astronomischen und kosmologischen Wissenschaft, sondern aus ihm resultiert auch eine systematische Verschränkung von epistemologischen Strukturen und ästhetischen Strategien. Als entscheidender Schritt dieser Entwicklung, so die These der Untersuchung, lässt sich die Frühromantik verstehen: Sie insistiert zum einen auf dem Bruch mit der tradierten Vorstellung des Kosmos und entdeckt darin die Möglichkeitsbedingung moderner Subjektivität. Zum anderen hat sie die Erfahrung einer solchen Destruktion noch zur Voraussetzung ihrer eigenen Poetik.

Dem gängigen Urteil zufolge gilt die Romantik indes als Inbegriff eines ebenso naiven wie hoffnungslosen Wunsches nach der Restitution einer imaginierten vorgängigen Einheit. Sie bezieht demnach ihre Ideen vornehmlich aus einer sehnsüchtigen Innenschau, dem harmonietrunkenen Einheitswunsch und der einsamen Schwärmerei bei sternklarer Nacht. Obgleich die Forschung der vergangenen Jahre ein weitaus differenzierteres Bild der Frühromantik und insbesondere ihrer naturwissenschaftlichen und subjekttheoretischen Studien zeichnen konnte, herrscht diese Vorstellung noch immer vor. Die vorliegende Untersuchung legt ihren Akzent dagegen auf das bis heute vernachlässigte kritische Profil der Frühromantik. Anhand der Lektüre ebenso zentraler wie wirkmächtiger Schriften der Frühromantik soll eine Problematik freigelegt werden, die das kosmologische Denken der Zeit betrifft. Der Bruch mit dem Kosmos, so die Annahme, wird von der Frühromantik erstmalig und sehr genau in seiner vollen Konsequenz registriert und bildet sodann den Ausgangspunkt von Studien, theoretischen Überlegungen und poetischen Texten. Im Gegensatz zur landläufigen Auffassung erzeugt die Frühromantik entschiedene Irritationen in den gängigen Formtraditionen, mit denen sie auf diese elementare Veränderung reagiert. Im Folgenden soll daher nicht erneut der vermeintliche Einheitswunsch der romantischen Theorie im Zentrum stehen, sondern vielmehr die hiervon abweichende und weitaus beunruhigendere Kehrseite: nämlich die neu entdeckte Abgründigkeit des eigenen Ich sowie die aus dieser Entdeckung resultierenden Konsequenzen für das kosmologische Denken. Mit der Analyse des Zusammenhangs von Kosmos und Subjektivität wird nicht nur ein 
grundlegendes geistesgeschichtliches Ereignis systematisch bestimmt, es gelangt damit auch eine bislang kaum berücksichtigte Seite der Romantik in den Blick.

Die entscheidenden Entwürfe einer wissenschaftlichen, philosophischen sowie poetischen Auseinandersetzung mit dem Zusammenhang von Kosmos und Subjektivität sind vorrangig im Umkreis der Jenaer Frühromantik zu verorten. So ist Jena in dieser Zeit Zentrum nicht nur philosophischer und literarischer, sondern auch naturwissenschaftlicher Entwicklungen und Entdeckungen. Die Jenaer Frühromantik zeichnet sich dadurch aus, dass sie ihre Poetik stets im Wechselbezug zu astronomischen, chemischen, physikalischen und transzendentalphilosophischen Studien erarbeitet. Materiale Grundlage der vorliegenden Untersuchung sind die theoretischen und literarischen Projekte, wie sie im Umkreis der Zeitschrift Athenäum in den Jahren zwischen 1796/97 und 1803/04 entstehen. Die Protagonisten der romantischen ,Konstellation', die im Folgenden im Zentrum stehen, sind Friedrich von Hardenberg (genannt Novalis), Bonaventura (wahrscheinlich Ernst August Friedrich Klingemann), Friedrich Wilhelm Joseph Schelling, Friedrich Schlegel und Friedrich Daniel Ernst Schleiermacher. ${ }^{1}$ Auch wenn dieser Konstellation fraglos weitere Personen hinzugefügt werden könnten, erweist sich eine Fokussierung auf die genannten im Hinblick auf die Problematisierung des Zusammenhangs von Kosmos und Subjektivität als zielführend. Gemeinsam ist den untersuchten frühromantischen Entwürfen, dass sie sich interdisziplinär mit den leitenden Wissenschaften und der Erschließung neuer Darstellungstechniken auseinandersetzen. Sie nehmen die Herausforderungen der modernen Wissenschaft an und bemühen sich um eine eigene, romantische Kosmologie. Eine Kosmologie indes, die allein unter umgekehrten Vorzeichen funktioniert.

Die Untersuchung wird sich auf die genannten Jahre und den überschaubaren Personenkreis der Frühromantik begrenzen. Textkorpus und Gegenstand der Analyse stehen also in einer denkbar großen Disproportion. Dies ist durchaus beabsichtigt, denn erst in der detaillierten Perspektive werden die Konturen des hier fokussierten Problems deutlich, welche sich in einem grobperspektivischen Überblick allzu leicht verlieren würden. Mit der Verabschiedung des Kosmos und dem simultanen (und diesen Abschied verursachenden) Auftreten der Subjektivität ist der Kern der romantischen Diskussion aufgerufen. Diesem irreduziblen Bruch und dem kritischen Potenzial der frühromantischen Philosophie und Poetik gilt das Interesse der Analyse, nicht aber den nachfolgenden Entwicklungen der deutlich sich abgrenzenden Spätromantik. Zwar lassen sich originäre Theorien und Dich-

1 Als Erster spricht Dieter Henrich in Bezug auf die Zeit der nachkantischen Philosophie von einem grundlegenden „konstellationalen Prozeß“ (Dieter Henrich, „Konstellationsforschung zur klassischen deutschen Philosophie. Motiv - Ergebnis - Probleme - Perspektiven - Begriffsbildung", in: Konstellationsforschung, hrsg. von Martin Mulsow und Marcelo Stamm, Frankfurt am Main 2005, S. 15-31, hier: S. 27). Der konstellative - und damit bereits ,kosmologisch situierte - Charakter dieses Gefüges wurde in der Forschung bereits ausgiebig diskutiert. Vgl. Manfred Frank, ,Unendliche Annäherung. Die Anfänge der philosophischen Frühromantik, Frankfurt am Main 1997; Dieter Henrich, Konstellationen. Probleme und Debatten am Ursprung der idealistischen Philosophie (1789-1795), Stuttgart 1991. 
tungen der Romantik, die sich mit dem Kosmos auseinandersetzen, mindestens noch bis zur Mitte des 19. Jahrhunderts ausmachen. Thema der Analyse ist jedoch weder die phantasmatische Restaurierung eines bürgerlich-behaglichen Kosmos etwa bei Achim von Arnim, Clemens Brentano, Joseph von Eichendorff und Alexander von Humboldt -, noch sind die Aktualisierungen des Bruches mit der kosmischen Ordnung, wie sie sich bei Stéphane Mallarmé, Samuel Beckett, Paul Celan und bis in die Gegenwart hinein finden, Gegenstand der Untersuchung.

Aus wissenspoetologischer Perspektive fragt die Untersuchung nach den Darstellungsverfahren kosmologischen Wissens. Wissenspoetologie ist ein Analyseverfahren, welches ästhetische Entscheidungen von Wissensordnungen untersucht, „sie interessiert sich demnach für die Regeln und Verfahren, nach denen sich ein Äußerungszusammenhang ausbildet und abschließt und die Darstellungen diktiert, in denen er seine performative Kraft sichert". ${ }^{2}$ Mit dieser Ausrichtung kann sowohl dem universalwissenschaftlichen Anspruch als auch dem epochalen Selbstverständnis der verhandelten Schriften Rechnung getragen werden. Die wechselseitigen Reflexionsbezüge von Kosmos und Subjektivität um 1800 sind bislang weder systematisch analysiert, noch ist deren exemplarische Bedeutung für eines der wichtigsten wissenschaftlichen Paradigmen der Neuzeit, nämlich den Kopernikanismus, gesehen worden. So hat die ,klassische Wissenschaftsgeschichte zwar bereits einen Übergang von einer Theorie der Welt oder des Kosmos hin zum Begriff des Universums registriert. Dabei wurde jedoch der unveräußerliche Selbstbezug nicht erkannt, der erst Subjektivität im Unterschied zum Kosmos auftreten lässt, zudem sind Rolle und Funktion der literarischen Verfahren, rhetorischen Techniken und performativen Strategien in dieser Entwicklung nicht hinreichend berücksichtigt worden. Das in der Kosmologie verhandelte Wissen ist aber nicht jenseitig von diesen Darstellungstechniken zu denken. Wissen ist vielmehr angewiesen auf historisch variable Strategien, Vollzugsformen und Entscheidungen, die seiner Darstellung zugrunde liegen.

Eine wissenspoetologische Untersuchung nimmt die Gestaltung, die Formatierung und die ästhetischen Strategien der untersuchten Texte in den Fokus. In Bezug auf die Theorie und Poetik der Romantik ist das von besonderer Relevanz: Die Romantik zeichnet sich nämlich nicht zuletzt dadurch aus, dass sie den Modus der ästhetischen Entscheidungen in ihren Werken reflektiert. Dies betrifft, wie im Folgenden zu zeigen sein wird, nicht allein astronomische und kosmologische $\mathrm{Zu}$ sammenhänge, sondern im Wechselverhältnis damit auch transzendentalphilosophische Fragen. Die Untersuchung wird auf die entscheidenden Argumente, taktischen Einsätze und poetischen Verfahren und Strukturen in den diversen romantischen Texten aufmerksam machen, die aus dem Bruch mit dem Kosmos

2 Joseph Vogl, „Einleitung“, in: Poetologien des Wissens um 1800, hrsg. von Joseph Vogl, München 1999, S. 7-19, hier: S. 13. Vgl. auch Nicolas Pethes, „Poetik/Wissen“, in: Romantische Wissenspoetik. Die Künste und die Wissenschaften um 1800, hrsg. von Gabriele Brandstetter und Gerhard Neumann, Würzburg 2004, S. 341-372; Jacques Rancière, Die Namen der Geschichte. Versuch einer Poetik des Wissens, Frankfurt am Main 1994, S. 17. 
und der Krise der Kosmologie resultieren. Wissen und seine Darstellung, so weist die wissenspoetologische Analyse nach, stehen in einer strengen Wechselbeziehung und werden einander alleine in einer systematischen Betrachtung transparent.

Bevor die bisherigen Erträge der Forschung zu der angeführten Problematik genauer diskutiert werden, sollen zunächst die beiden zentralen Begriffe - Kosmos und Subjektivität - in einem knappen, historischen Überblick skizziert werden.

Der Begriff des Kosmos (vom griechischen kósmos sich herleitend) ist durch eine lange und wechselvolle Geschichte bestimmt. ${ }^{3}$ In der griechischen Antike bezieht er sich zunächst auf die militärische Ordnung und wird erst im sechsten vorchristlichen Jahrhundert durch Anaximander für die Bezeichnung der Welt als einer geordneten sowie harmonischen Einheit gebräuchlich. Als Kosmologie ist eine vollständige und geschlossene Theorie des Kosmos definiert, wie sie seit der Antike angestrebt wird. Ihr kommt eine doppelte Aufgabe zu: Zum einen bestimmt sie den Kosmos als eine einheitliche und harmonische Ordnung, als ,wohlgeordneten Schmuck' also, wie er aus antiker Tradition überliefert ist. Der Kosmos weist danach eine sowohl in mathematischer wie in ästhetischer Perspektive harmonische Struktur auf. Zum anderen will die Kosmologie diese Ordnung in eine ebenso einheitliche wie wohlgeordnete Theorie übertragen. Spätestens mit Platon ist der Kosmos ästhetisch konzipiert; so ist das Weltganze ihm zufolge von einem Demiurgen nach sowohl mathematischen wie harmonischen Gesetzen konstruiert. Auch Aristoteles legt Wert auf die harmonische Struktur eines kugelförmigen Kosmos, außerhalb dessen für ihn nichts existiert. Bis in das vom Ordo-Denken geprägte Mittelalter bleibt die aristotelische Kosmologie die vorherrschende.

Erst mit der beginnenden Neuzeit kommt es zu den großen Umbrüchen, von denen die kopernikanische Wende vom geo- zum heliozentrischen Weltbild der grundlegendste ist. Mit ihnen verändert sich nicht nur die physikalische Raumvorstellung, sondern auch die Theorie der Natur fundamental. ${ }^{4}$ Nach der These Alexandre Koyrés erfährt der Kosmos mit den wissensgeschichtlichen Entwicklungen der Neuzeit eine „Zerstörung“ und wird substituiert „durch ein grenzenloses und sogar unendliches Universum, das durch die Identität seiner fundamentalen Be-

3 Eine Begriffsgeschichte kann im Rahmen dieser Untersuchung nur skizziert werden. Vgl. für detailliertere Ausführungen Gerhard Börner, Kosmologie, Frankfurt am Main 2002; Matthias Gatzemeier u. a., Art. „Kosmos, Antike-Neuzeit“, in: Historisches Wörterbuch der Philosophie [HWPh], 12 Bde., hrsg. von Joachim Ritter, Karlfried Gründer und Gottfried Gabriel, Basel u. a. 1971-2007, Bd. 4, Sp. 1167-1176; David Furley, „Kosmologie“, in: Das Wissen der Griechen. Eine Enzyklopädie, hrsg. von Jacques Brunschwig und Geoffrey Lloyd, München 2000, S. 304321; Bernulf Kanitscheider, Kosmologie. Geschichte und Systematik in philosophischer Perspektive, Stuttgart 1984; Walther Kranz, „Kosmos“, in: Archiv für Begriffsgeschichte 2 (1955), S. 7-266.

4 Die umfangreiche Forschung dazu kann nicht im Einzelnen diskutiert werden, es sei verwiesen auf die grundlegenden Arbeiten u. a. von Ladina Bezzola Lambert, Imagining the Unimaginable. The Poetics of Early Modern Astronomy, Amsterdam/New York 2002; Alexandre Koyré, Von der geschlossenen Welt zum unendlichen Universum, Frankfurt am Main 2008; Thomas S. Kuhn, The Copernican Revolution. Planetary Astronomy in the Development of Western Thought, Cambridge 1957; Stephen Shaphin, Die wissenschaftliche Revolution, Frankfurt am Main 1998. 
standteile und Gesetze zusammengehalten wird und in dem alle diese Bestandteile auf derselben Stufe des Seins stehen". 5 Allerdings berufen sich noch Kopernikus und Galilei auf die einheitliche Vorstellung des Kosmos, die erst mit den kantischen Antinomien gänzlich aufgegeben wird. Daher, so weist Hans Blumenberg nach, lässt sich der Übergang vom geozentrischen zum heliozentrischen Weltbild noch als eine Rettung mittelalterlicher Vorstellungen und damit auch des Kosmos verstehen. ${ }^{6}$

Die für die Neuzeit bestimmende Mechanisierung des Kosmos setzt mit der mathematischen Reformulierung aller natürlichen Bewegungen ein, welche Ausgangspunkt der Forschung Galileis sind. ${ }^{7}$ Mit Galileis teleskopischen Beobachtungen der Sterne und Planeten beginnt die Zeit der mediengestützten Astronomie. Sie löst die bis zur Antike zurückreichende Tradition des von Blumenberg so bezeichneten „Sichtbarkeitspostulat $[s]$ “8 ab. Nicht mehr die bloßen Sinne gelten von nun an als Garant der Wahrheit, sondern einzig die perspektivische Konstruktion und auf Daten gestützte Beobachtungen bezeugen veritable Sichtbarkeiten. Die Suspendierung des "Sichtbarkeitspostulats" in der Astronomie der Neuzeit führt Hans Blumenberg zufolge zu einem Anwachsen der Bedeutung des Imaginären. Das Subjekt wird in dieser Entwicklung zunehmend auf sich selbst aufmerksam: „Das ins Weite und Fremde treibende und getriebene Subjekt wird sich auffällig und sperrig in dem Maße, wie es sich in Raum und Zeit zu entgehen scheint".? Damit dringt nicht nur das Subjekt in die astronomischen Beobachtungsvorgänge ein, es wird vielmehr noch selbst zum Beobachtungsproblem, und zwar aufgrund seines Selbstbezugs.

Ihren größten Triumph erfährt die mathematisch-physikalische Naturtheorie 1686 mit Isaac Newtons Philosophiae Naturalis Principia Mathematica. ${ }^{10}$ Mit diesem Werk legt Newton den Grundstein der klassischen Mechanik, welche bis ins späte 19. Jahrhundert unumstößlich bleibt. Besondere Relevanz für die allgemeine Physik und Kosmologie besitzt das von Newton eingeführte Gravitationsgesetz: Mit der universal wirkenden Gravitationskraft lassen sich die Bewegungsabläufe fallender Körper beschreiben, zudem werden diese Gesetze auf die Umläufe der planetarischen Bahnen übertragen. Anziehungs- und Abstoßungskraft als wechsel-

5 Koyré, Von der geschlossenen Welt zum unendlichen Universum, S. 12.

6 Vgl. Hans Blumenberg, „Kosmos und System. Aus der Genesis der kopernikanischen Welt“, in: Studium Generale 10.2 (1957), S. 61 - 80, hier: S. 74 f.

7 Galileo Galilei, Schriften, Briefe, Dokumente, hrsg. von Anna Mudry, Wiesbaden 2005. Zur Metaphorisierung des Kosmos als machina vgl. u. a. Hans Blumenberg, „Organische und mechanische Hintergrundmetaphorik“, in: ders., Paradigmen zu einer Metaphorologie, Frankfurt am Main 1998, S. 92-110; René Descartes, Discours de la méthode, hrsg. u. übers. von Christian Wohlers, Hamburg 1997, AT XI 226; Nikolaus Kopernikus, De revolutionibus orbium caelestium. Textkritische Ausgabe, hrsg. von Franz Zeller und Karl Zeller, München 1949, S. 5; Eduard Dijksterhuis, Die Mechanisierung des Weltbildes, Berlin/New York 1983.

8 Hans Blumenberg, Die Genesis der kopernikanischen Welt, Frankfurt am Main 1981, S. 731.

9 Ebd., S. 722.

10 Isaac Newton, The Principia. Mathematical Principles of Natural Philosophy. A New Translation, Berkeley 1999. 
wirkende Grundkräfte werden im weiteren Verlauf des 18. Jahrhunderts zu dem bestimmenden Erklärungsschema astronomischer Naturbeschreibungen. Dabei ist es der frühe Immanuel Kant, der die Relevanz der Abstoßungskraft herausstellt.

Mit der deutlichen Verbesserung von Mess- und Beobachtungsinstrumenten, den neuen Messtechniken der Spektral- und Parallaxenanalyse, den mathematischen Verfahren der Newton'schen Mechanik und der Probabilistik sowie der revolutionären Theorie der Gravitationsphysik entwickelt die Astronomie des 18. Jahrhunderts das Selbstverständnis einer Art Leitwissenschaft. Entscheidend hierfür ist auch der Ausbau der Infrastruktur, erlaubt sie doch eine verbesserte Kommunikation und Distribution der von den Sternwarten regelmäßig erfassten Messdaten. Mitte des 18. Jahrhunderts beschreibt der britische Astronom Thomas Wright of Durham das Sonnensystem erstmalig als Teil eines größeren Umlaufsystems. Hans Blumenberg sieht darin einen entscheidenden Schritt in der Entwicklung der modernen Kosmologie: „Die zwei Jahrhunderte seit dem Erscheinen des Hauptwerkes von Kopernikus rücken jetzt zu einer Einheit zusammen: in dieser Zeit ist trotz der Entdeckungen mittels des Fernrohrs für das Gesamtbild des Universums nichts Entscheidendes mehr geschehen. Das Jahr 1750 bezeichnet die Wende mit der Original Theory or new Hypothesis of the Universe von Thomas Wright ${ }^{11}$ Mittels neuartiger Spiegelteleskope deckt Wright eine optische Täuschung auf und kann die Milchstraße anhand astronomischer Beobachtung nicht mehr als einen hellen Nebelschweif, sondern als einen galaktischen Komplex klassifizieren, in dem das irdische Sonnensystem nur einen winzigen Teil ausmacht. Die Milchstraße besitzt seinen Messungen zufolge nicht etwa eine lineare, sondern eine zirkuläre Struktur, die in sich rotiert. Damit wird die antike Idee eines äußeren Fixsternhimmels obsolet - sämtliche Sterne werden mittels dieser Bemessungen nun als Teil von stetig rotierenden Sonnensystemen und Galaxien erkannt. Johann Heinrich Lambert macht diese Vorstellung von Milchstraßensystemen zum Aus-

11 Blumenberg, Die Genesis der kopernikanischen Welt, S. 617. Der Einsatz von Riesenteleskopen, wie sie Wilhelm Herschel verwendet, führt zudem im weiteren Verlauf des 19. Jahrhunderts zur Entstehung der Disziplin der Astrophysik. Diese neuartige Disziplin gelangt auf empirischer Basis zu Exaktheitsgraden, wie sie bis dahin nur bei physikalischen Experimenten irdischer Naturphänomene bekannt waren. Vgl. dazu ebd., S. 675 ff.; Lorraine Daston und Elizabeth Lunbeck (Hrsg.), Histories of Scientific Observation, Chicago/London 2011; Simon Schaffer, „The Phoenix of Nature. Fire and Evolutionary Cosmology in Wright and Kant", in: Journal for the History of Astronomy 9 (1978), S. 180-200; Thomas Wright, An Original Theory or New Hypothesis of the Universe, Founded upon the Laws of Nature, Cambridge 2014. Richard Panek resümiert dazu: „In der Beziehung zwischen dem Teleskop und unserem Verständnis der Dimensionen des Universums spiegelt sich in vielerlei Hinsicht die Geschichte der Moderne wider" (Richard Panek, Das Auge Gottes. Das Teleskop und die lange Entdeckung der Unendlichkeit, Stuttgart 2004, S. 11). Für die hier behandelte Zeit lässt sich noch eine Verstärkung derselben Entwicklung nachweisen, so spricht Florian Welle gar von einer „Fernrohrmanie“ der Goethezeit (Florian Welle, Der irdische Blick durch das Fernrohr. Literarische Wahrnehmungsexperimente vom 17. bis zum 20. Jahrhundert, Würzburg 2009, S. 93). Für Hannah Arendt kündigt sich dieser Prozess bereits mit der Erfindung des Teleskops an, die bei ihr wesentlichen Anteil an der Entwicklung der Neuzeit hat (Hannah Arendt, Vita activa oder vom tätigen Leben, München 1981, S. 244 f.). 
gangspunkt seiner Cosmologischen Briefe. ${ }^{12}$ Darin geht er jedoch zusätzlich von der spekulativen Annahme eines äußersten Rotationszentrums aus, um welches das gesamte Universum kreist. ${ }^{13}$

Einen weiteren entscheidenden Schritt in der Entwicklung der Astronomie im 18. Jahrhundert markiert der Einsatz von Wahrscheinlichkeitsrechnung und Statistik innerhalb der Disziplin. Beide Methoden wirken den Abweichungen oder Aberrationen der Beobachtung entgegen und entwickeln sich zunehmend zu einem methodischen Verfahren der astronomischen Beschreibung. ${ }^{14}$ So entwirft etwa Pierre-Simon Laplace erstmalig eine statistisch ermittelte Berechnung des Sonnensystems. ${ }^{15}$ Mittels dieser Methoden beginnt eine Ablösung der rationalen metaphysischen Kosmologie durch eine empirische Astronomie. Sie wird von der schriftlichen Erfassung und Kartografierung in wissenschaftlichen Kompendien begleitet. In ganz Europa erscheinen weit verbreitete vielbändige Werke zur Beschreibung des Himmels und seiner Phänomene. Unter den Autoren sind namhafte Politiker, Ökonomen und Philosophen. ${ }^{16}$ Adam Smith, Begründer der klassischen Nationalökonomie, verfasst eine eigene Astronomiegeschichte. ${ }^{17}$ Die darin konzipierte invisible hand, welche ihm zufolge den Umlauf der Planeten sichert, überträgt Smith später auf den Bereich der Ökonomie. ${ }^{18}$ Und auch Jean-Sylvain Bailly, der in der

12 Johann Heinrich Lambert, Cosmologische Briefe über die Einrichtung des Weltbanes, Augsburg 1761. Blumenberg stellt das Differenzmerkmal zur Kosmologie Kants derweil unter dem Aspekt der Zeitlichkeit heraus (Blumenberg, Die Genesis der kopernikanischen Welt, S. 670). Kants geschichtliche Bestimmung erweist sich ihm zufolge als die letztlich durchsetzungsfähigere.

13 Lambert wählt zur Darstellung seiner Kosmologie eine Reihe von Briefen, die eine inszenierte Fortsetzung der Dialogen über die Mehrheit der Welten von Fontenelle vorstellen. Es kündigt sich damit eine Sequenzierung der Kosmologie an, die nicht mehr als geschlossene auftritt, sondern den naturgeschichtlichen Prozess ins eigene episodische Narrativ integriert. Vgl. Bernard Le Bovier de Fontenelle, Dialogen über die Mehrheit der Welten, Berlin 1789; ders., Entretiens sur la pluralité des mondes, Paris 1686.

14 Zum Komplex von Wahrscheinlichkeitsrechnung und Astronomie vgl. u. a. Frédérique AitTouati, Fictions of the Cosmos. Science and Literature in the Seventeenth Century, Chicago 2011; Lorraine Daston, Classical Probability in the Enlightenment, Princeton/New York 1988.

15 Pierre-Simon Laplace, Darstellung des Weltsystems, hrsg. u. übers. von Manfred Jacobi, Frankfurt am Main 2008. Vgl. zu Laplace u. a. Jörn Henrich, Die Fixierung des modernen Wissenschaftsideals durch Laplace, Berlin 2010; Ilya Prigogine und Isabelle Stengers, Dialog mit der Natur. Neue Wege des naturwissenschaftlichen Denkens, München/Zürich 1980, bes. S. 81-84.

16 Zum Verhältnis von Astronomie und Aufklärung vgl. Peter-André Alt, „Kopernikanische Lektionen. Zur Topik des Himmels in der Literatur der Aufklärung“, in: Germanisch-Romanische Monatsschrift 48 (1998), S. 141-165; Rainer Baasner, Das Lob der Sternkunst. Astronomie in der deutschen Aufklärung, Göttingen 1987; Paul Feyerabend, Naturphilosophie, Frankfurt am Main 2002, S. 271-320.

17 Adam Smith, „History of Astronomy“, in: ders., Essays on Philosophical Subjects, hrsg. von John Cameron Bryce und William P. D. Wightman, Oxford 1980, S. 1-93.

18 Adam Smith, An Inquiry into the Nature and Causes of the Wealth of Nations, London 1776. Vgl. zum Motiv der invisble hand daneben auch Stefan Andriopoulos, „The Invisible Hand. Supernatural Agency in Political Economy and the Gothic Novel", in: English Literary History 66 (1999), S. 739-758; Ralf Klausnitzer, „Unsichtbare Fäden, unsichtbare Hand. Ideengeschichte und Figuration eines Metaphernkomplexes", in: Begriffe, Metaphern und Imaginationen in Philosophie und Wissenschaftsgeschichte, hrsg. von Lutz Danneberg, Carlos Spoerhase und Dirk Werle, Wiesbaden 2009, S. 145-176; Joseph Vogl, Das Gespenst des Kapitals, Zürich 2010, S. 39 ff. 
Zeit der Französischen Revolution das Amt des ersten Pariser Bürgermeisters innehat, ist professioneller Astronom. ${ }^{19}$

Neben den technischen Errungenschaften und der verbesserten Wissensdistribution sind für die Hochphase der Astronomie und Kosmologie die Schriften von Immanuel Kant, Johann Heinrich Lambert und Pierre-Simon Laplace verantwortlich, die umfassende Darstellungen des Kosmos liefern. ${ }^{20}$ Im Rekurs auf die Gravitationslehre Newtons gelingt den Autoren eine physikalische Reformulierung aller natürlichen Bewegung sowie eine Erklärung der Entstehung und Entwicklung des Kosmos. Mit der geschichtlichen Organisation der Kosmologie geht indes eine entscheidende Transformation einher: So verstand sich die Kosmologie der kopernikanischen Tradition stets noch als Rettung einer krisenhaften, geschlossenen, ,alten' Kosmologie. Erst mit der anthropologischen Wende um die Mitte des 18. Jahrhunderts wandelt sich dieser teleologische Systemkosmos zur Vorstellung eines Lebensraums, in dem die gesicherte und bevorzugte Stellung des Menschen im Kosmos grundlegend infrage gestellt ist. ${ }^{21}$ Der Kosmos als harmonisches Systemprinzip wird mit Kants Nachweis der Antinomien der rationalen Kosmologie endgültig verabschiedet, wie im Folgenden noch zu zeigen ist. Die Disziplin der Kosmologie, deren traditioneller Gegenstand der einheitliche und gesetzesmäßige Kosmos ist, verliert fortan unausweichlich an Bedeutung. Die Epistemologie sichert nunmehr als philosophische Erstdisziplin die Gesetzlichkeit der Erfahrung. Gleichzeitig leiten die statistische Mechanik und die Bestimmung der thermodynamischen Gesetze eine Krise der Kosmologie ein. Mit der probabilistischen Wende in der Thermodynamik zeigt sich zunehmend, dass statistische Regelmäßigkeiten auf Makroebene grundlose Ursachen auf der Ebene der kleinsten Teilchen haben. Eine einheitliche und harmonische Theorie der Welt erweist sich damit als unmöglich. So ist bereits gegen Ende des 19. Jahrhunderts die einstige Leitdisziplin Kosmologie durch ihre eigene Tochterdisziplin Astrophysik substituiert.

Anders als der Begriff des Kosmos reicht das Konzept der Subjektivität nicht bis in die Antike zurück. Vielmehr muss die Instanz des Subjekts als ein neuzeitliches Phänomen (und zwar noch in Abgrenzung zum antiken hypokeimenon) verstanden werden. Subjektivität lässt sich zunächst bestimmen als das grundsätzliche Vermögen, intentionale Akte im Rückbezug auf das eigene Ich, das heißt im Wissen um

19 Bekannt wird Bailly durch die Bestimmung der Umlaufbahn des Halleyschen Kometen sowie durch seine Histoire de l'astronomie (Jean-Sylvain Bailly, Histoire de l'astronomie, 5 Bde., Paris 1775-1787). Vgl. dazu u. a. David Aubin, „On the Cosmopolitics of Astronomy in 19th-century Paris“, in: Astroculture. Dialogues of Cosmology and Cosmopolitanism in Media and Culture, hrsg. von Sonja Neef und Henri Sussmann, Paderborn 2013, S. 61-84.

20 Pierre-Simon Laplace, Euvres complètes, hrsg. von der Académie des sciences, 14 Bde., Paris 1878-1912; deutsch: ders., Darstellung des Weltsystems. Die in der Epoche bekanntesten Schriften zur Astronomie sind die Exposition du Système du Monde von Simon de Laplace sowie das Lehrbuch der Astronomie von Abel Bürja, das zwischen 1794 und 1805 erscheint (Abel Bürja, Lehrbuch der Astronomie, Berlin 1794).

21 Vgl. Jörn Garber und Heinz Thoma, „Vorwort“, in: Zwischen Empirisierung und Konstruktionsleistung. Anthropologie im 18. Jahrhundert, hrsg. von dens., Tübingen 2004, S. I-X, hier: S. VII f. 
sich selbst zu vollbringen. Ein Subjekt ist damit, wie Christoph Menke es formuliert, „ein Wesen, dessen Bezüge auf anderes - Objekte wie andere Subjekte wesentlich dadurch bestimmt sind, daß sie von einem Selbstbezug ,begleitet sind“". ${ }^{22}$ Der historische Einsatzpunkt von Subjektivität ist das berühmte cartesianische cogito. Wenngleich bei Descartes das Subjekt noch nicht in einem modernen Sinne auftaucht - und man mit Étienne Balibar diesbezüglich gar von einer kantischen ,Fehlinterpretation' sprechen kann -, wird das Ich mit den Meditationes dennoch zum wesentlichen Erkenntnisprinzip der Philosophie der Neuzeit und bestimmende Instanz der modernen Entwicklung philosophischer, politischer sowie rechtlicher Diskurse. ${ }^{23}$

Es ist wiederum Immanuel Kant, der mit seiner Kritik der reinen Vernunft die bekannte kopernikanische Wende der Subjekttheorie einleitet: Das Selbstbewusstsein ist nun insofern der gesicherte Ausgangspunkt der Philosophie, als es als transzendentale Einheit der Apperzeption einen Anspruch auf objektive Erkenntnis zu sichern vermag. ${ }^{24}$ Die transzendentale Einheit der Apperzeption ermöglicht Objektivität mittels des Ich, das aber selbst nicht Gegenstand der Erfahrung sein kann, weil es diese überhaupt erst konstituiert. Für die nachkantische Philosophie wird die Frage nach der Möglichkeit eines solchen gesicherten Ausgangspunktes zum wesentlichen Gegenstand. Den entscheidenden Lösungsvorschlag liefert zuerst Johann Gottlieb Fichte in seiner Grundlage der gesamten Wissenschaftslehre von $1794 .{ }^{25}$ Seinem Verständnis nach ist das reine Selbstbewusstsein nur möglich als eine Anschauung. Diese jeder sinnlichen Erfahrung vorangehende intellektuelle Anschauung ist der absolute Ausgangspunkt jeglicher Form von Erkenntnis. ${ }^{26}$ Anschauendes Subjekt und angeschautes Objekt sind in diesem Verhältnis identisch. Das angeschaute Ich muss in der Konstruktion aber erst im Ich konstruiert werden, um Teil seiner Anschauung werden zu können. Es geht also erneut das Resultat der eigenen Erklärung voraus. Die Möglichkeit einer unbedingten gesicherten Er-

22 Christoph Menke, Art. „Subjektivität“, in: Ästhetische Grundbegriffe. Historisches Wörterbuch in sieben Bänden, Bd. 5, hrsg. von Karlheinz Barck u. a., Stuttgart/Weimar 2003, S. 734-787, hier: S. 734.

23 Vgl. Étienne Balibar, Barbara Cassin und Alain de Libra, Art. „Sujet“, in: Vocabulaire Européen des Philosophies, hrsg. von Barbara Cassin, Paris 2004, Sp. 1233-1253, hier: Sp. 1246; ders., „Subjection and Subjectivation“, in: Supposing the Subject, hrsg. von Joan Copjec, London/New York 1994, S. 1-16; René Descartes, Meditationes de Prima Philosophia. Meditationen über die Grundlagen der Philosophie, hrsg. u. übers. von Christian Wohlers, Hamburg 1992; Brigitte Kible, Art. „Subjekt“, in: Historisches Wörterbuch der Philosophie, Bd. 10, Sp. 373-400, hier: Sp. 379.

24 Immanuel Kant, Kritik der reinen Vernunft, in: ders., Werkausgabe, Bd. IV u. V, hrsg. von Wilhelm Weischedel, Frankfurt am Main 1974 [KrV], B 132-133.

25 Johann Gottlieb Fichte, Grundlage der gesamten Wissenschaftslehre, in: Sämmtliche Werke, Bd. I/1, hrsg. von Immanuel Hermann Fichte, Berlin 1971. Vgl. dazu vor allem die Arbeiten Henrichs: Dieter Henrich, Fichtes ursprüngliche Einsicht, Frankfurt am Main 1966; ders., Grundlegung aus dem Ich. Untersuchungen zur Vorgeschichte des Idealismus. Tübingen - Jena 1790-1794, 2 Bde., Frankfurt am Main 2004; ders., Konstellationen. Probleme und Debatten am Ursprung der idealistischen Philosophie (1789-1795), Stuttgart 1991.

26 Fichte, Grundlage der gesamten Wissenschaftslehre, S. $228 \mathrm{ff}$. 
kenntnis vom Ich ist damit weiterhin ungeklärt und steht auch im Zentrum der Diskussionen in der Frühromantik. ${ }^{27}$

Ein wesentliches Ziel der frühromantischen Philosophie besteht darin, die Wissenschaftslehre Fichtes mittels der spinozistischen Substanzphilosophie auf eine objektive Ebene zu heben. ${ }^{28}$ Der als unvollständig angesehene idealistische Ansatz Fichtes soll also eine Synthese mit der realistischen Position Spinozas erfahren. Eine der für die frühromantische Philosophie einflussreichsten Schriften ist dabei die Ethik in geometrischer Ordnung dargestellt des Baruch de Spinoza. ${ }^{29}$ Die Natur wird darin nicht nur als Produkt einer göttlichen Wirkung (wie etwa bei Platon) dargestellt, sondern als diese Wirkung selbst. Es gibt für Spinoza keinen hierzu jenseitigen Bereich, in dem die göttliche potentia noch nicht aktualisiert wäre. Gott ist in der Ethik Spinozas als eine unendliche Substanz konzipiert, als „ein unbedingt unendliches Seiendes, d. h. eine Substanz, die aus unendlich vielen Attributen besteht, von denen jedes eine ewige und unendliche Essenz ausdrückt". ${ }^{30}$ Spinozas Entwurf bietet ein Universum reiner Immanenz auf, in dem alle Dinge Manifestationen einer Substanz sind. Diese Substanz ist ,Ursache ihrer selbst', causa sui, sie ist nur durch sich selbst bedingt und realisiert sich aus eigenem Antrieb, das heißt, dass ihre „Essenz Existenz einschließt ${ }^{\text {“ }}{ }^{31}$ Geist und Natur sind zwar aus der Perspektive des endlichen Denkens schlechthin verschieden, erweisen sich jedoch als Attribute einer einzigen Substanz. Mit der Annahme einer singulären Substanz, deren Unbegrenztheit sich dem begrenzten reflexiven Denken entzieht, ist der Einsatz eines philosophischen Realismus angezeigt. Der Hinweis Spinozas auf eine einzige Substanz als jenseitiger Grund für das reflexive Denken ist ein wesentlicher

27 Vgl. dazu grundlegend u. a. Manfred Frank, Einführung in die frühromantische Ästhetik. Vorlesungen, Frankfurt am Main 1989; ders., Das Problem, Zeit in der deutschen Romantik. Zeitbewußtsein und Bewußtsein von Zeitlichkeit in der frühromantischen Philosophie und in Tiecks Dichtung, Paderborn 1990; ders., Unendliche Annäherung; Birgit Rehme-Iffert, Skepsis und Enthusiasmus. Friedrich Schlegels philosophischer Grundgedanke zwischen 1796 und 1805, Würzburg 2001.

28 Auch wenn bekannt ist, dass der Spinozismus grundlegende Bedeutung für das Selbstverständnis der frühromantischen Generation besitzt, wird die theoretische Verortung Spinozas von der literaturwissenschaftlichen Forschung noch immer kaum zur Kenntnis genommen. Zum Stellenwert des Spinozismus in der nachkantischen Philosophie vgl. David Bell, Spinoza in Germany from 1670 to the Age of Goethe, London 1984; Eckart Förster, Die 25 Jahre der Philosophie. Eine systematische Rekonstruktion, Frankfurt am Main 2011, S. 89 f.; Hermann Timm, Gott und die Freiheit, Bd. 1: Die Spinozarenaissance, Frankfurt am Main 1974 und Johannes Brachtendorf, „Absolutheit und Subjektivität. Zur Spinoza-Rezeption des deutschen Idealismus“, in: System and Context. Early Romantic and Early Idealistic Constellations. New Athenaeum, hrsg. von Rolf Ahlers, Lewiston 2004, S. 191-278. Brachtendorf untersucht unter anderem die Rezeption durch Hölderlin und Schelling.

29 Baruch de Spinoza, Opera, Bd. 2, hrsg. von Carl Gebhardt, Heidelberg 1925; deutsch: ders., Ethik in geometrischer Ordnung dargestellt, in: Sämtliche Werke, Bd. 2, hrsg. von Wolfgang Bartuschat, Hamburg 2010. Die 1677 posthum erschienene Abhandlung findet eine starke Rezeption mittels der Briefe Jacobis, welche indes mit der Absicht verfasst sind, eine Widerlegung dieser Lehre zu leisten. Vgl. Friedrich Heinrich Jacobi, Über die Lehre des Spinoza in Briefen an den Herrn Moses Mendelssohn, in: ders., Werke, Bd. I/1, hrsg. von Klaus Hammacher und Irmgard Maria Piske, Hamburg 1998.

30 Spinoza, Opera, Bd. 2, S. 45 f.

31 Ebd., S. 45. 
Impuls für die Romantik und bildet sogleich den realistischen Gegenpol zum Idealismus Fichtes. In einer polemischen Passage seiner Reden Über die Religion sieht Friedrich Schleiermacher diesbezüglich mit „dem vollendeten und gerundeten Idealismus", für den Fichte paradigmatisch einsteht, die Gefahr aufkommen, dass jener „das Universum vernichten“" werde, ,indem er es zu bilden scheint“ ${ }^{32}$ Der adressierte Idealismus verkennt demnach die wesentliche Funktion von Subjektivität, die in der Einsicht in die Unmöglichkeit von Totalität besteht. Daher wäre es Schleiermacher zufolge nötig, „das Gegengewicht“ zu halten, um einen „höheren Realismus ahnden“" zu lassen..$^{33}$

Im Anschluss an die kantische Philosophie ist es wesentliches Anliegen der romantischen, ein Prinzip auszumachen, welches den Gesamtzusammenhang möglicher Erkenntnisse begründet. Dieses Prinzip wird in der philosophischen Diskussion der Zeit als Unbedingtes oder Absolutes gefasst. Gesichertes Wissen, das keine bloße Spekulation ist, sondern dem Realität zukommt, kann es - so das Postulat der Romantik - nur im Rückgriff auf ein selbst unbedingtes Begründungsmoment geben. Im Widerstreit von empirischer und spekulativer Wissenskonzeption gilt es somit zwei Anforderungen zu entsprechen: Zum einen muss die spekulative Erkenntnis ein Moment der empirischen Mitteilbarkeit aufweisen, um überhaupt zur Realität zu gelangen. Zum anderen muss auch die empirische Erkenntnis einen absoluten Bedingungsgrund für ihr Wissen angeben. Während Fichte und in dessen Nachfolge Schelling dieses Unbedingte im absoluten Ich in einer intellektuellen Anschauung erkennen, schlagen Friedrich Schlegel und Novalis ein negatives oder kritisches Prinzip vor: Es sei kein Absolutes als Bedingungsgrund alles Wissens einsehbar, sondern nur der unbedingte Anspruch auf ein Wissen. Das negative Verhältnis dieser Ansätze ergibt aus romantischer Perspektive einen systematischen Zusammenhang, der sich nur als dialektischer Prozess realisiert. ${ }^{34}$ Die von Schlegel erkannte Bedeutung des Widerspruchs für das Erkennen findet sich in seiner Konzeption des Wechselerweises oder Wechselgrundsatzes expliziert. ${ }^{35}$ Dem Absoluten kommt somit innerhalb der Philosophie eine systemati-

32 Friedrich Schleiermacher, Über die Religion. Reden an die Gebildeten unter ihren Verächtern, hrsg. von Hans-Joachim Rothert, Hamburg 1958, S. 54.

33 Ebd. Andreas Arndt bestimmt die Aufgabe von Schleiermachers, höherem Realismus' dahingehend, dass er „den Gehalt dessen, was Spinoza unter der Begriffsform und daher (nach Schleiermachers Auffassung) widersprüchlich gedacht hatte, auf unbegriffliche Weise voraussetzt und gleichwohl für das endliche Begreifen als zwingend erweist" (Andreas Arndt, Friedrich Schleiermacher als Philosoph, Berlin/New York 2013, S. 97).

34 Vgl. dazu Arndt: „Die Einheit von System und Geschichte realisiert sich als widersprüchliche Einheit von ,Selbstschöpfung" und "Selbstvernichtung' jeweiliger Systemzustände" (Andreas Arndt, „Friedrich Schlegels dialektischer Systembegriff“, in: System und Systemkritik um 1800, hrsg. von Christian Danz und Jürgen Stolzenberg, Hamburg 2011, S. 287-300, hier: S. 299).

35 Vgl. dazu Manfred Frank, „Wechselgrundsatz. Friedrich Schlegels philosophischer Ausgangspunkt", in: Zeitschrift für philosophische Forschung 50 (1996), S. 26-50; ders., ,Unendliche Annäherung', S. 862-886; Guido Naschert, „Friedrich Schlegel über Wechselerweis und Ironie, Teil 1“, in: Athenäum. Jahrbuch für Romantik 6 (1996), S. 47-90; ders., „Friedrich Schlegel über Wechselerweis und Ironie, Teil 2", in: Athenäum. Jahrbuch für Romantik 7 (1997), S. 11-36; Rehme-Iffert, Skepsis und Enthusiasmus, bes. S. 14-82. 
sche Funktion zu, die jedoch streng negativ auf den Bereich der Empirie wirkt. Vermittlung mit Objektivität als Prozess der subjektiven Erkenntnis vollzieht sich dem romantischen Verständnis nach allein geschichtlich als ein kritisches Verfahren bestimmter Negation oder Skepsis.

Die Romantik begreift Subjektivität also nicht nur als ein die Einheit der Erfahrung garantierendes Ordnungsprinzip, sondern insistiert emphatisch auf ihrer Funktion als operative Leerstelle, auf der modernen Erfahrung von Subjektivität als konstitutivem und irreduziblem Bruch. So spricht Manfred Frank von einem

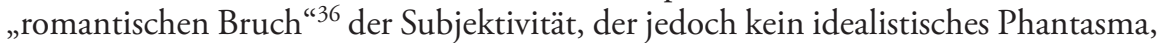
sondern objektives Resultat der modernen Wissensgeschichte sei: „Der Zustand der zersplitterten Welt ist keineswegs eine Negativphantasie der Romantik, sondern ein realer Effekt des analytischen Geistes der Neuzeit und insbesondere der Aufklärung ". ${ }^{37}$ Subjektivität markiert demzufolge die Systemstelle ontologischer Diskontinuität. Damit wird das Subjekt innerhalb der Romantik Gegenstand von grundlegenden Darstellungsfragen. In der Reflexion auf die eigene Identität zeigt sich nämlich deren Grund stets als jenseitig, und die Darstellung dieser Relation bleibt das einzige erkenntnisleitende Residual philosophischer Arbeit. Herbert Uerlings zufolge wird das Konzept des Darstellens in der Frühromantik „zum Oberbegriff für eine unendliche Produktivität des Subjekts, die, nicht nur im Bereich des Wissens, immer auch Selbsterzeugung und Selbst-Darstellung bedeutet ${ }^{\text {“. }}{ }^{38}$ Die Begründung des Ich verlangt nach seiner Darstellung, die zugleich „einen wechselseitigen Transfer zwischen Naturwissenschaften [...] und Künsten "veranlasst. ${ }^{39}$ Andreas Arndt fasst diesen romantischen Ansatz der Selbstbegründung wie folgt: „In der Reflexion aber setzt das Ich den Gehalt außer sich: es verlässt das Identische, um es darzustellen. Diese Entfremdung ist notwendiges Produkt des Bewußtseins, das eben darum kein Wissen der ursprünglichen Identität zulässt, denn es ist als Wissen unausweichlich in dieser Objektivation befangen ". ${ }^{40}$ In der Darstellung gelangt ein anderes Wissen zum Vorschein, das nicht auf die reflexiven Gehalte reduziert werden kann. Damit wird die Ästhetik dieser Zeit, so Christoph Menke, „zum zentralen Medium für das Interesse des Subjekts an sich selbst - für die Bestimmung und

36 Manfred Frank, „Das ,fragmentarische Universum“ der Romantik“, in: Fragment und Totalität, hrsg. von Christiaan Lucas Hart Nibbrig und Lucien Dällenbach, Frankfurt am Main 1984, S. 212-224, hier: S. 215. Vgl. etwa auch Rehme-Iffert, Skepsis und Enthusiasmus, S. 52; Christian Iber, „Frühromantische Subjektkritik“, in: Fichte und die Romantik. Hölderlin, Schelling, Hegel und die späte Wissenschaftslehre, hrsg. von Wolfgang H. Schrader, Amsterdam 1997, S. 111-126.

37 Frank, „Das ,fragmentarische Universum“ der Romantik“, S. 220.

38 Herbert Uerlings, „Darstellen. Zu einem Problemzusammenhang bei Novalis“, in: Romantische Wissenspoetik, S. 373-391, hier: S. 390. Vgl. dazu weiter: „Ausgehend von der in den Fichte-Studien gewonnenen Einsicht, daß sich das (absolute) Ich als unendlich darstellendes setzt [...], wird das Konzept der Darstellung für Hardenberg zentral. Darstellung bleibt bei ihm bezogen auf das Identische und ist in diesem Sinne Construction" (ebd.). Vgl. dazu auch Claudia Albes, „Einleitung", in: Darstellbarkeit. Zu einem ästhetisch-philosophischen Problem um 1800, hrsg. von ders. und Christiane Frey, Würzburg 2003, S. 9-28, hier: S. 16.

39 Uerlings, „Darstellen“, S. 390.

40 Arndt, Friedrich Schleiermacher als Philosoph, S. 44. 
Entfaltung von Subjektivität“. ${ }^{41}$ Dabei markiert die Romantik den historischen Zeitpunkt des „Ende[s] der ästhetischen Subjektivität" - Novalis und Friedrich Schlegel liefern die entscheidenden theoretischen Entwürfe, mit denen Ästhetik und Subjektivität als einander erschließende Pole verstanden sind. ${ }^{42}$

In der Forschung hat die Bedeutung des Kosmos und der Kosmologie für die romantische Theorie bislang kaum Beachtung erfahren. Dies ist zum einen deswegen überraschend, weil den Wissenschaften der ,Sternkunde' allgemein eine Nähe zu romantischen Interessen nachgesagt wird. Zum anderen gab es in den vergangenen Jahrzehnten die gegenläufige Tendenz einer intensiven Auseinandersetzung mit der romantischen Wissenschaftstheorie und -praxis, in deren Fokus (vor dem Hintergrund der Wissenspoetologie) meist eine ,Poetisierung der Wissenschaften im Umkreis der Romantik stand. Dabei lässt sich eine übereinstimmende Sichtweise auf die Romantik konstatieren: So wird in diesem Zusammenhang einhellig die „epistemologische[...] Umbruchssituation“ der Zeit um 1800 anerkannt, die romantische Philosophie und Dichtung hingegen als „Versuch“ interpretiert, „noch einmal die Einheit der Welt im Namen eines Ganzen der ,Natur' wiederherzustellen", wie beispielsweise Herbert Uerlings schreibt. ${ }^{43}$ Zweifelsohne beansprucht das Naturwissen um 1800 - etwa in der Gestalt der Elektrizitätslehre oder der Chemie -, mit wissenschaftlichen Argumenten noch einmal die Idee einer Einheit von Mikrokosmos und Makrokosmos zu rechtfertigen. ${ }^{44}$ Jedoch muss die Romantik als der entscheidende Schritt der epistemischen Umbruchsituation verstanden werden, die sich weniger nur durch ein Festhalten an der potenziellen Einheit der Welt auszeichnet, als vielmehr dadurch, dass sie Subjektivität als radikalen Bruch mit einer solchen Vorstellung entdeckt.

Maßgebliche Vorarbeit für das Verständnis des kosmologischen Denkens der Romantik leistete Hans Blumenberg mit der Genesis der kopernikanischen Welt. Bei seiner Untersuchung der Konzeptions- und Nachgeschichte der Theorie des Himmels von Kant kommt er auf wenigen Seiten auf Friedrich Schlegel und Schelling zu sprechen. Die Darstellung findet jedoch bei aller gedanklichen Präzision und beispiellosen Sachkenntnis auf einem für den Diskussionszusammenhang der philosophischen Romantik allzu komprimierten Raum statt. Sie bietet bloß knappe Einblicke in die Positionen Schlegels und Schellings, während der naturwissenschaftliche Fokus der Romantik sowie die damit in Wechselbeziehung stehenden poetischen Arbeiten keine Beachtung finden. Die Studien Manfred Franks bieten

41 Menke, „Subjektivität“, S. 735.

42 Ebd., S. 766. Ein „ästhetisches Subjekt“ ist, so Menke, zweifach bestimmt: „erstens als Wahrheitsgehalt des Schönen und zweitens als Grund wie Ziel der nicht-ästhetischen Gestalt des Subjekts“ (ebd.).

43 Herbert Uerlings, „Novalis und die Wissenschaften. Forschungsstand und Perspektiven“, in: ders. (Hrsg.), Novalis und die Wissenschaften, Tübingen 1997, S. 1-20, hier: S. 2.

44 Vgl. hierfür etwa beispielhaft die Studien von Michael Gamper, Elektropoetologie. Fiktionen der Elektrizität 1740-1870, Göttingen 2009, bes. S. 134-151; Benjamin Specht, Physik als Kunst. Die Poetisierung der Elektrizität um 1800, Berlin 2010, bes. S. 253-266. 
besonders für den Aspekt der Subjekttheorie nach wie vor einige der differenziertesten Interpretationen der Frühromantik. Der Bezug zum Kosmos ist darin zwar kaum präsent, es finden sich aber bereits in seinem Beitrag zum Fragmentarischen Universum der Romantik zielführende Bestimmungen des Problems. ${ }^{45}$ Allerdings dienen die Begriffe der Welt und des Universums dort allein als metaphorische Referenten und besitzen keine wissensgeschichtliche Qualität. Die naturwissenschaftlichen Studien von Novalis etwa können jedoch nicht nur als Nebenprodukte seiner theoretischen und poetischen Arbeit angesehen werden, sondern die Problematik der Kosmologie ist für ihn maßgeblicher wissenschaftlicher Gegenstand sowie Ausgangspunkt für eigene, romantische Darstellungsfragen. Andreas Arndt verweist in seinem Aufsatz Naturgesetze der menschlichen Bildung. Zum geschichtsphilosophischen Programm der Frühromantik knapp auf Schlegels „Programm einer an der Himmelsmechanik orientierten Geschichtswissenschaft", mit der dieser sich zum „Newton der Geschichte“ aufschwingen möchte. ${ }^{46}$ Arndt zufolge knüpft Schlegel mit seinem kosmologischen Geschichtskonzept „an einen Topos in der Literatur der 2. Hälfte des 18. Jh. [an], dessen Ursprung und Wege freilich keineswegs als geklärt gelten können “. ${ }^{47}$ Als weitere Wegbereiter dieses Topos nennt Arndt auch Johann Gottfried Herder, Wilhelm von Humboldt und Immanuel Kant, deren Bezugnahmen auf Kosmologie und Naturgeschichte in aller Kürze skizziert werden. Auf die Funktion der Kosmologie innerhalb der Frühromantik geht Arndt nicht ein, seine Analyse fokussiert den Bildungsbegriff Schlegels in seinem dezidiert historischen Verständnis. Bei Ulrich Stadler findet sich eine für die Forschung wesentliche Einzeluntersuchung zur „poetische[n] Theorie der Fernröhre" von Novalis. ${ }^{48}$ Er stellt die epistemologische Bedeutung des Mediums Teleskop für die Romantik heraus und deckt weitreichende Bezüge innerhalb der Schriften von Novalis auf. Eine inhaltliche Auseinandersetzung mit der Kosmologie und Astronomie der Zeit wie auch der romantischen Theorie der Subjektivität bleibt bei Stadler jedoch aus. Ebenfalls belässt es das ansonsten umfangreiche Werk Wissenschaftswahrnehmung und -umsetzung im Kontext der deutschen Frühromantik von Erk F. Hansen bei beiläufigen Anmerkungen zu diesem Bereich. ${ }^{49}$ Hier findet sich immerhin eine anerkennende und in der Materialfülle beispielhafte Untersuchung etwa der Physik im Umkreis der Frühromantik, ohne jedoch deren Bezüge

45 Frank, „Das ,fragmentarische Universum“ der Romantik“, S. 212-224, bes. S. 220 ff.

46 Andreas Arndt, „Naturgesetze der menschlichen Bildung. Zum geschichtsphilosophischen Programm der Frühromantik“, in: Die Zukunft des Wissens. XVIII. Deutscher Kongreß für Philosophie Konstanz 1999, hrsg. von Jürgen Mittelstraß, Konstanz 1999, S. 1141-1148, hier: S. 1141.

47 Ebd., S. $1141 \mathrm{f}$.

48 Ulrich Stadler, „Arbeit am Instrument I. Die Herstellung vermittelter Unmittelbarkeit oder Novalis' ,Poetische Theorie der Fernröhre“", in: ders., Der technisierte Blick. Optische Instrumente und der Status von Literatur. Ein kulturhistorisches Museum, Würzburg 2003, S. 144-155.

49 Erk F. Hansen, Wissenschaftswahrnehmung und -umsetzung im Kontext der deutschen Frühromantik. Zeitgenössische Naturwissenschaft und Philosophie im Werk Friedrich von Hardenbergs (Novalis), Frankfurt am Main 1992, bes. S. 365-372. 
zur Astronomie und Kosmologie hinreichend zu erschließen. ${ }^{50}$ Ebenso werden die poetischen Schriften in dieser Untersuchung nicht näher betrachtet.

Eine Konjunktur der Nachtthematik lässt sich in den diversen Kulturgeschichten der vergangenen Jahre ausmachen. ${ }^{51}$ Allerdings ist es den historiografischen Studien gemeinsam, dass sie von der frühromantischen Theorie fast gänzlich absehen. Am ausführlichsten wird die romantische Naturforschung bei Heinz-Gerhard Friese verfolgt. Hier dient Schuberts „Real-Metapher der Nachtseite der Natur und des Menschen " in historischer Perspektive als Indiz einer Entdeckung unbewusster Strukturen. ${ }^{52}$ Diese Lesart ist sicherlich zutreffend, sie verzichtet jedoch auf die philosophisch-begriffliche wie auch darstellungstheoretisch-poetische Problematisierung der Kosmologie und des Kosmos. Mit der Fixierung der Nacht als transhistorischer Metapher ist zudem der problematische Umbruch, der sich in der Destruktion des Kosmos um 1800 vollzieht, nicht zu erfassen. Einen übergreifenden geschichtlichen Zugang wählt auch die Arbeit Tiefer als der Tag gedacht. Eine Kulturgeschichte der Nacht von Elisabeth Bronfen. ${ }^{53}$ Sie zeichnet sich in besonderer Weise dadurch aus, dass sie den spezifischen Bruch der Zeit berücksichtigt und auch auf Hegels Frühschriften näher eingeht. Die Bedeutung der Subjektivität als selbstbezügliche Negativität und ,nächtliches' Prinzip wird herausgestellt, jedoch der wissensgeschichtliche Aspekt dieser Verkehrung nicht berücksichtigt. ${ }^{54}$ Der Frühromantik (in Gestalt von Novalis) gesteht Bronfen wohl eine „innere geistige Nacht" zu, betrachtet jedoch nicht ihre negative Funktion hierbei, sondern bedient vielmehr erneut die Vorstellung von Novalis als Einheitsdichter. ${ }^{55}$ Barbara Hunfeld untersucht in ihrer Studie Blick ins All anhand von Brockes, Jean Paul, Goethe und Stifter literarische Zugriffe auf astronomische Blickkonstellationen, explizit sind bei ihr aber „[n]icht die Inhalte der Welt- oder Selbstwahrnehmung [...] Gegenstand der [...] Untersuchung, sondern deren semiotische Konstitution".56 Aus zeichentheoretischer Perspektive fragt sie nach den konstellativen Konstitutionsprinzipien von Texten und macht damit den ,Kosmos der Zeichen` zum Gegenstand

50 Erk F. Hansen stellt in seiner Untersuchung die Physik als Disziplin der Astronomie voran, bemerkt jedoch im Hinblick auf deren Binnendifferenzierung anerkennend: „Durch das Wirken von Physikern wie Galilei, Kepler und vor allem durch die ,Philosophiae naturalis principia mathematica' I. Newtons von 1686 hatte sich die Mechanik (einschließlich der Himmelsmechanik) zum disziplinären Kernstück der klassischen Physik vor 1800 sowie zu einer ,Modelltheorie` der Physik überhaupt entwickelt" (ebd., S. 54).

$51 \mathrm{Zu}$ den bekannten Werken allein im deutschsprachigen Raum gehören Elisabeth Bronfen, Tiefer als der Tag gedacht. Eine Kulturgeschichte der Nacht, München 2008; Ernst Peter Fischer, Durch die Nacht. Eine Naturgeschichte der Dunkelheit, München 2015; Heinz-Gerhard Friese, Die Ästhetik der Nacht. Eine Kulturgeschichte, Reinbek 2011.

52 Friese, Die Ästhetik der Nacht, S. 663.

53 Bronfen, Tiefer als der Tag gedacht.

54 Ebd., S. 104. Vgl. auch: „Das Ich, dessen Geist sich im Denken entfaltet, ist somit grundsätzlich durch dieses Stück Nacht, das der Mensch in sich trägt, gebildet" (ebd., S. 106).

55 Ebd., S. 77. Vgl. auch ebd., S. 80 f.

56 Barbara Hunfeld, Der Blick ins All. Reflexionen des Kosmos der Zeichen bei Brockes, Jean Paul, Goethe und Stifter, Tübingen 2004, S. 2. 
ihrer Studie, der jenseits einer Subjektkonstitution zu verorten wäre und ihrer These zufolge sowohl den Kosmos als auch Subjektivität substituiert. ${ }^{57}$

Ein zunehmendes Interesse von Seiten der Forschung lässt sich in den letzten Jahren für die naturphilosophischen und kosmologischen Arbeiten Schellings registrieren. Hier seien vor allem die Arbeiten von Marie-Luise Heuser erwähnt, die Schellings Vorarbeiten für die Konzeptionen der Selbstorganisation erstmalig reaktualisiert. ${ }^{58}$ In vergleichbarer Weise und unter Bezug auf Gilles Deleuze bemüht sich Ian Hamilton Grant um eine Aktualisierung der frühen naturphilosophischen Schriften Schellings. ${ }^{59}$ Tanja Gloyana widmet sich der Kosmoskonzeption Schellings, in der sie ein geschichtlich sich entfaltendes „System unseres Wissens" ausmacht. ${ }^{60}$ Schellings für kosmologische Fragen wesentliches Gespräch Bruno wird jedoch von der Schelling-Forschung weitestgehend ignoriert. Dabei kommen im ästhetisch formierten Gespräch wissenspoetische Momente zum Tragen, die sich nicht nur für eine literaturwissenschaftliche Analyse in besonderer Weise eignen, sondern darüber hinaus auch notwendiges Komplement der Schelling'schen Kosmologie sind.

Die vorliegende Studie wird ausgehend von den bisherigen Ergebnissen der Forschung ein eigenständiges Diskussionsfeld freilegen, das dem Problemzusammenhang von Kosmos und Subjektivität gewidmet ist. Eine Aufgabe neben der Betrachtung der ideen- und wissensgeschichtlichen Entwicklungen der Zeit wird es sein, die poetologischen Aspekte der behandelten Schriften zu untersuchen. Denn die dichterischen Entwürfe der Frühromantik stehen nicht jenseits der subjekttheoretischen und naturwissenschaftlichen Schriften, sondern müssen mit ihnen zusammen und als ein sich wechselweise ausbildendes, strategisches Ensemble betrachtet werden.

Die Untersuchung wird wie folgt vorgehen: Erstens wird sie die Schritte nachzeichnen, die zur Destruktion des Kosmos führen und alternative Modelle auf den Plan rufen (Kap. „I. Romantische Kosmologie“). Dabei sollen die Entwicklungen innerhalb der Kosmologie sowie die Bedeutung der kantischen Antinomien herausgestellt werden. Die Frühschrift Immanuel Kants zur Allgemeinen Naturgeschichte, in der der Kosmos erstmals in einer modernen wissenschaftlichen Genese beschrieben

57 Vgl. ebd., S. 208. Dabei erweist sich der semiotische Kosmos jedoch nicht als Substitut, sondern vielmehr als Begleiterscheinung der Suspendierung des Kosmos, die mit der Ablösung der Kosmologie durch Erkenntnistheorie und Astronomie einhergeht. Die von Hunfeld konstatierte „produktive[...], immanente[...] Unabschließbarkeit“ des „Kosmos der Zeichen“ ließe sich durchaus als mit der Programmatik der romantischen Universalpoesie korrespondierend bestimmen, jedoch mit dem entscheidenden Vermerk, dass diese Produktion der Zeichen eben aus einem spezifischen Zusammenhang von Kosmos und Subjektivität resultiert (ebd., S. 210).

58 Vgl. Marie-Luise Heuser, Die Produktivität der Natur. Schellings Naturphilosophie und das neue Paradigma der Selbstorganisation in den Naturwissenschaften, Berlin 1986; dies. und Wilhelm G. Jacobs (Hrsg.), Schelling und die Selbstorganisation. Neue Forschungsperspektiven, Berlin 1994.

59 Ian Hamilton Grant, Philosophy of Nature after Schelling, London u. a. 2006.

60 Tanja Gloyana, Kosmos und System. Schellings Weg in die Philosophie (Schellingiana 15), Stuttgart 2002, S. 5. 
ist, eignet sich in besonderer Weise, um den Kenntnisstand der Kosmologie und Kosmogonie zu dokumentieren (Kap. „Destruktion des Kosmos“). Mit den Antinomien der rationalen Kosmologie, die Kant in seiner Kritik der reinen Vernunft nachweist, steht die Kosmologie als einheitliche Theorie der Natur vor unauflöslichen Aporien. Der irreduzible Bruch, den das Subjekt im Verständnis von der Totalität des Kosmos erzeugt, bedeutet die endgültige Verabschiedung des Kosmos im Sinne einer positiven Seinsordnung.

Friedrich Schlegels Vorlesungen zur Transcendentalphilosophie bieten ein Modell, das diese Problematik in ihren Konsequenzen erfasst und sich von dem aus den Antinomien resultierenden Bruch her denkt (Kap. „Bewusstsein des Unendlichen"). Das bedeutet keine Absage an die kritische Philosophie, sondern es ist erklärtes Ziel Schlegels, eine Theorie der Welt zu projektieren, die von den neuen Voraussetzungen ausgeht. So zeichnet sich die anvisierte Theorie durch ihr Verfahren der Selbstreflexivität aus, das stets auf eine unveräußerliche Inkonsistenz verweist. Das Individuum dient dieser Theorie als zentrale Instanz, an der sich die Unendlichkeit des Universums manifestiert. Die romantische Kosmologie bleibt aus diesem Grunde nicht nur fragmentarisch, sondern verweist als autoreferenzielle noch auf die eigene antinomische Struktur. Auch das frühromantische Programm der Universalpoesie erschließt sich dergestalt als kosmologisch angeleitet.

Anhand von Schellings Schrift Bruno wird beispielhaft eine nachkantische spekulative Kosmologie untersucht, seine frühen theoretischen Arbeiten werden ergänzend hinzugezogen (Kap. „Polyhymnios Schweigen“). Ziel ist es zu zeigen, dass eines der wesentlichen Anliegen des frühen Schelling eine restituierte Theorie der Natur oder Kosmologie ist. Es bedarf jedoch dezidiert ästhetischer Verfahren und Strategien, um die Natur in ihrer Dynamik für den endlichen Verstand erkennbar zu machen. Der Wechsel vom streng begrifflichen Diskurs hin zu einem poetisch markierten dient in Schellings Gespräch Bruno dazu, die Voraussetzungen und Konsequenzen einer nachkantischen, spekulativen Kosmologie immanent zu erproben. Die Parallelen sowie Differenzen zum Programm der romantischen Kosmologie werden dabei deutlich.

Zweitens fragt die Untersuchung nach den epistemischen Konsequenzen, die sich aus dem Bruch mit dem Kosmos ergeben (Kap. „II. Epistemischer Kopernikanismus"). Die Reden Schleiermachers Über die Religion stellen diesbezüglich das Modell eines genuinen Pluralismus vor: Die Aleatorik der astronomischen Erscheinungen und ihre Rückkopplung in der Beobachtung ermöglichen Handlungspotenziale, die stets auf eine Vielheit der Perspektiven angewiesen sind (Kap. „Vielheit der Perspektiven“). In einem fortlaufenden Prozessieren sind die Betrachter angehalten, sich auf wechselnde Positionen einzulassen. Ein solcher Pluralismus erlaubt den Verweis auf die dezidiert materialistische Tradition der Kosmologie im Anschluss an Lukrez.

Die Enzyklopädistik des Novalis bietet das Modell eines universal wechselwirkenden Nexus der Wissenschaften (Kap. „Universalnexus“). Dieser zeichnet sich durch die Reflexion auf seine eigene Brüchigkeit und eine dynamische Struktur im Sinne des epistemischen Kopernikanismus aus. Die Vernetzung sämtlicher Teildis- 
ziplinen entwirft Novalis in seinem Allgemeinen Brouillon. Ein dergestalt konzipierter Universalnexus findet im Universalkompendium der Enzyklopädie das spezifisch romantische Medium seiner Darstellung. Der Universalnexus soll jedoch keinen vorgängigen Kosmos abbilden, sondern muss vielmehr die Vernetzungen zwischen seinen Elementen überhaupt erst konstruieren. Das Verständnis einer fundamentalen Neuordnung ist dabei auch politisch konnotiert, wie anhand von Novalis' Modell der Kosmopolitik aufgezeigt wird. Dass der epistemische Kopernikanismus auch Auswirkungen auf die Romanpoetik der Romantik zeitigt, wir anhand der Lehrlinge zu Sais von Novalis nachgewiesen.

Drittens tritt mit dem angezeigten Bruch ein Drama des Subjekts zutage, dem sich die Arbeit in einem abschließenden Schritt widmet (Kap. „III. Inversion des Blicks"). Den differenziertesten Versuch einer romantischen Selbstbestimmung unternimmt Novalis in seinen Fichte-Studien. Seine ordo-inversus-Lehre hat die Fichte'sche Selbstbewusstseinsphilosophie zum Ausgang, die Novalis mit dem philosophischen Gegenpol der Lehre Spinozas synthetisiert (Kap. „Abfahrt ins Innere“). Der Konzeption einer inversen Reflexion zufolge ist das Gefühl des Ich nicht auf das Selbst begrenzt, sondern vielmehr ununterscheidbar von der vorgängigen einen Substanz. Daraus resultiert das Phantasma eines inneren Universums, dem sich Novalis in seinen Studien und Entwürfen widmet.

Eine literarische Verdichtung dieses Komplex findet sich in Novalis' Hymnen an die Nacht (Kap. „Unendliche Augen“). Der ordo inversus ist im Gedicht nachvollzogen und macht den eigenen kosmologischen Anspruch deutlich. Die Nacht der Hymnen ist weniger ein privatmythologischer Rückzugs- und Ursprungsort, sondern vielmehr unweigerliches Signum eines irreduziblen Bruches. In dieser Ambivalenz gelingt es dem Zyklus, einen radikalen Kontextbruch zu vollziehen, der in letzter Konsequenz noch auf die vorherrschende Vorstellung von Geschichte zielt. Im Bruch mit der herkömmlichen Auffassung des Kosmos sowie dem geschichtlichen Kontinuum ereignet sich in den Hymnen eine Blickumkehr. Der Blick der Vergangenen der Geschichte ist demnach auf die Gegenwart gerichtet und ihre Sehnsucht ist es, welche die eigene bedingt.

Abschließender Untersuchungsgegenstand des Zusammenhangs von Kosmos und Subjektivität sind die (vermutlich von Ernst August Friedrich Klingemann verfassten) Nachtwachen von Bonaventura (Kap. „Grundlose Nacht“). Hier leitet eine rigorose Erzählhaltung die eigene Poetik an, die auf den strengen Bezug zum destruierten Kosmos referiert. Der Kosmos erweist sich als ein mortifizierter, das erzählende Ich erkennt sich erst im Unterschied zu einer solchen Ordnung. Subjektivität begreift sich somit allein im Ausschluss aus einer positiven Seinsordnung und in der Entdeckung ihres traumatischen Kerns. Das Subjekt der Nachtwachen ist darum eines, das erst im Durchgang des eigenen Phantasmas sich selbst konstituiert. 